\title{
sbtools: A Package Connecting R to Cloud-based Data for Collaborative Online Research
}

\author{
by Luke A Winslow, Scott Chamberlain, Alison P Appling and Jordan S Read
}

\begin{abstract}
The adoption of high-quality tools for collaboration and reproducibile research such as $\mathrm{R}$ and Github is becoming more common in many research fields. While Github and other version management systems are excellent resources, they were originally designed to handle code and scale poorly to large text-based or binary datasets. A number of scientific data repositories are coming online and are often focused on dataset archival and publication. To handle collaborative workflows using large scientific datasets, there is increasing need to connect cloud-based online data storage to $R$. In this article, we describe how the new $R$ package sbtools enables direct access to the advanced online data functionality provided by ScienceBase, the U.S. Geological Survey's online scientific data storage platform.
\end{abstract}

\section{Introduction}

Cloud data storage platforms can be a powerful tool for research collaboration, distributed computing, and data publication. However, while browser-based graphical interfaces make these platforms accessible to a wide audience, there is also a need for scripted data access and manipulation so that researchers can capture data provenance and create reproducible analyses. For example, Figshare (Singh, 2011), DataOne (Michener et al., 2012), Dataverse (King, 2007) and CKAN (Winn, 2013) are all research data sharing platforms that are gaining use in different research fields. For each of these platforms, the community has released (Boettiger et al.; Leeper, 2013; Chamberlain) or is developing (Jones et al., 2013) R packages to streamline the storage and access to archived data.

These existing projects primarily focus on creating more useful, open, and accessible end products of research, but data- and code-intensive collaborative projects increasingly need collaborative solutions for data storage, sharing, and updating not just at the end of the project, but throughout the full project lifecycle. Github is increasingly used to collaborate around data products (Gandrud, 2013b), but does not scale well to the distribution of large datasets (Delcambre, 2013) and does not include metadata or queries beyond free-text search. Furthermore, most data archive platforms like DataOne and Dataverse focus on academic research projects and do not support some of the unique needs of federal research. For example, many government institutions require an archived copy of released data to be stored and available through federally operated websites. Third party storage providers are insufficient.

To address future scientific data sharing and archival challenges, the U.S. Geological Survey (USGS) created ScienceBase (https://www. sciencebase.gov/). This platform is designed to support the full project data lifecycle and has seen rapid adoption with USGS researchers and collaborators. ScienceBase supports the storage and access of large files and datasets. It allows data to be stored with a user-configurable mixture of public and authenticated access and has been designed from the beginning with first-class RESTful web interfaces to support robust API access. Items on ScienceBase can have hierarchical relationships, facilitating the organization of complex or related data. It also supports a seamless transition from project development to data publication, focusing on searchable, accessible, well described datasets for public use and citation.

To expand the usefulness of the ScienceBase platform directly to $\mathrm{R}$ workflows, we have designed and implemented an $\mathrm{R}$ interface to ScienceBase called sbtools. This interface provides scripted $\mathrm{R}$ access to ScienceBase to manage metadata and data files, to search the catalog of datasets, and to view and modify data in formats familiar to $\mathrm{R}$ users. Here we describe several features of ScienceBase, and how we have implemented the $\mathrm{R}$ interface to make them accessible and useful from $\mathrm{R}$.

\section{USGS ScienceBase}

To facilitate and encourage data sharing and dissemination, the U.S. Geological Survey has created ScienceBase, an online collaborative scientific data platform (Figure 1). ScienceBase is targeted for use by USGS researchers, their collaborators, and the end users of reviewed and released USGS data products. ScienceBase has four key elements to support collaborative data workflows: 1) Data and metadata cataloging and hosting with options for private, controlled access and fully public sharing 
of data and metadata. 2) Central search and data discovery for both data hosted on ScienceBase and externally hosted data. 3) Full web-service support for all core functionality, including standards-based access to specific data types (e.g. geospatial datasets). 4) Research community catalogs to enable the organization of data along collaborative group and organizational boundaries.

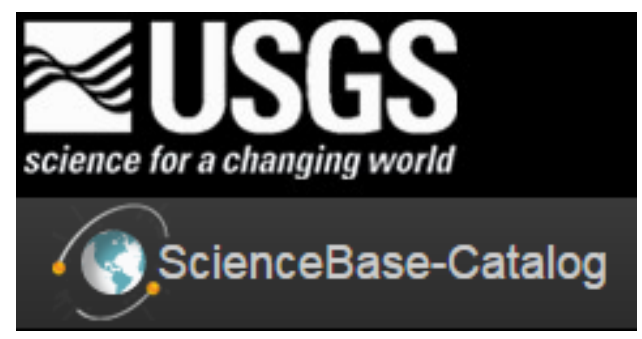

Figure 1: The ScienceBase platform logo

ScienceBase users store data in 'items', where each item is a flexible representation of a dataset and its metadata. The dataset component of an item can be one or more data files in any format or simply an item with descriptive metadata linking a dataset hosted on an external repository. This supports indexing of legacy datasets that are hosted in well known locations (for example, the USGS National Hydrography Dataset). Items are organized into a tree hierarchy (much like the structure of files and folders on a hard drive). This allows data to be intuitively organized by the institution, collaborative group, and/or individual to whom the data belong. At the same time, items can also be assigned identifying tags for rapid search and data discovery across the full ScienceBase catalog.

Search and management of ScienceBase data items can be accomplished through both graphical and scripted interfaces. Manual search, data upload/download, and metadata editing are possible through the ScienceBase website. Automated access to all of these functions is supported by robust RESTful web services and a documented API. The ScienceBase code and infrastructure setup is available upon request from the ScienceBase team <sciencebase@usgs.gov>. Further information and details can be found in the online ScienceBase Documentation.

\section{The sbtools package}

In creating sbtools, our goal was to allow complete access to the ScienceBase web service API in a flexible, lightweight $\mathrm{R}$ package. The package imports a minimal number of external packages to support core functionality. More advanced data access (e.g. geospatial web services) is supported through suggested packages to keep the basic installation requirements minimal for all platforms. Within R, sbtools is designed to keep end-user interactions simple despite the underlying complexity of many of the web service calls (e.g. authentication).

Below, we describe briefly the core functions available in sbtools and discuss the unique features of ScienceBase that sbtools provides $\mathrm{R}$ users.

\section{Data access API}

The data access functionality of sbtools makes it easy to access any public item. Every item in ScienceBase has a unique identifier that can be used for direct access to the item and its associated data and metadata. A lightweight representation of this information is created in $\mathrm{R}$ with the item_get function.

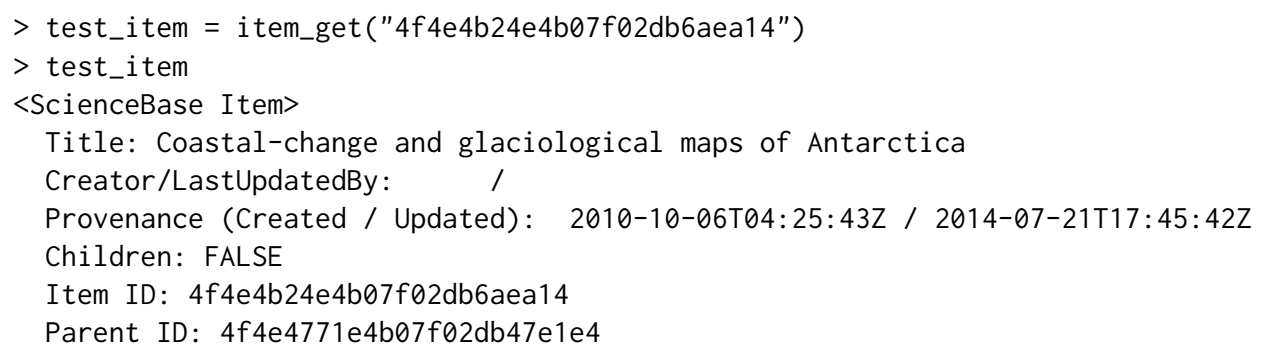


This representation is defined by sbtools as an "sbi tem" object, which contains many fields and can be further inspected in the same way as a named list.

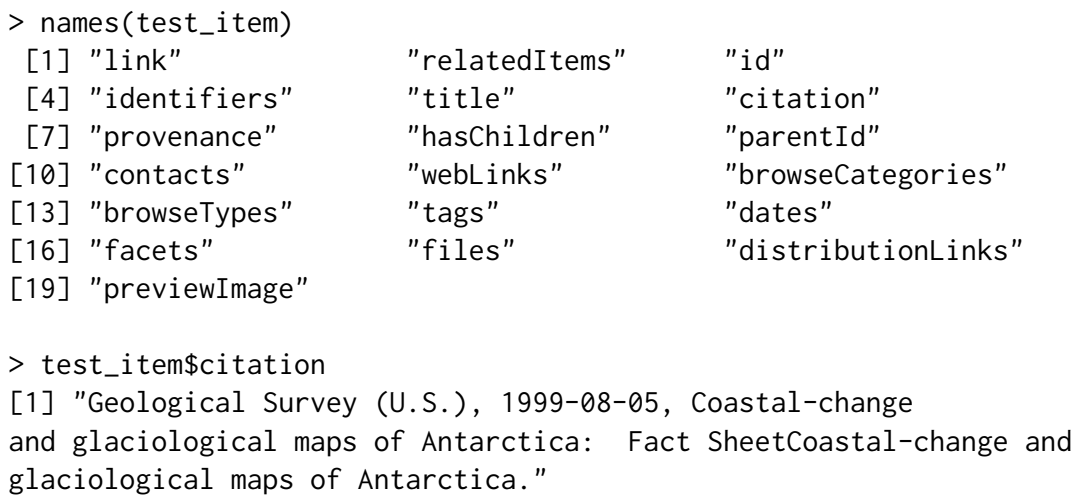

On ScienceBase, the hierarchical item tree dictates relationships between items; each item has one parent and potentially many children. sbtools allows the user to easily traverse this tree structure. Because ScienceBase allows users to define their internal organization, this heirarchy can take on different meanings for different projects, including conveying data provenance or spatial grouping.

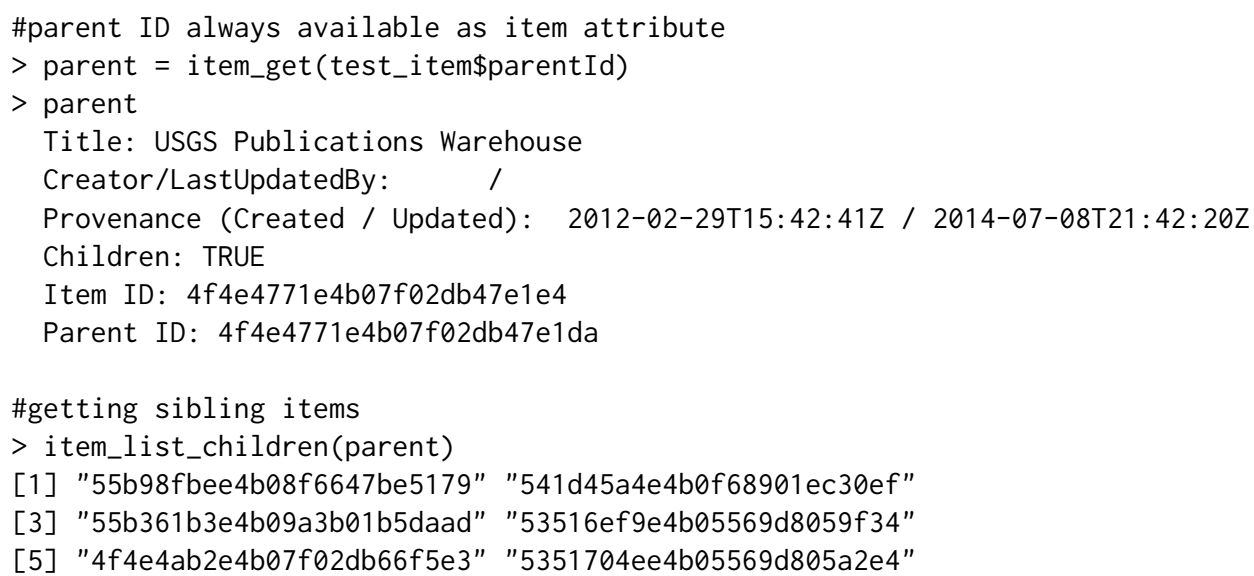

ScienceBase items may have data or metadata files attached to them. You can list and download attached files directly using sbtools.

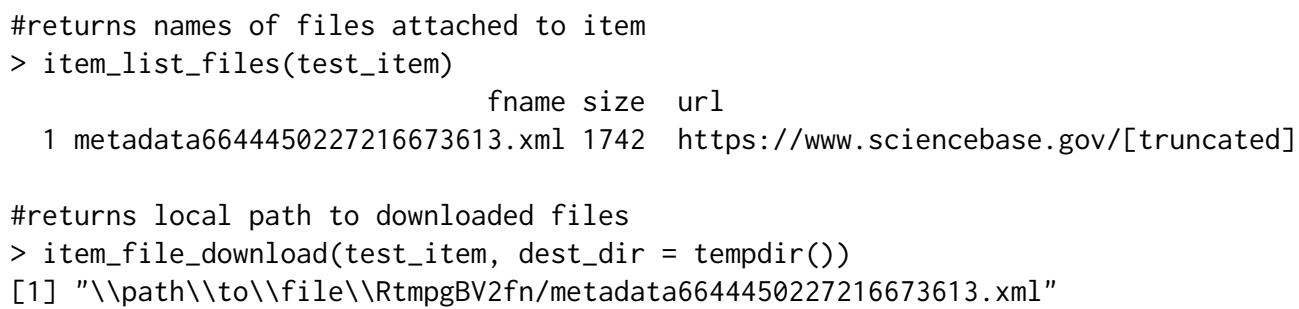

ScienceBase has special functionality for certain data types. One example is spatial data. When spatial data are uploaded to ScienceBase and appropriate metadata is included, they can be accessed using Open Geospatial Consortium (OGC) web services. sbtools includes functionality to access Web Feature Service (WFS) when available. Once retrieved, spatial data are stored as sp spatial objects, which are easily manipulated and visualized (Figure 2). Note: Some figure formatting details omitted from code below for simplicity. See demo (' figure_map_code ', package= 'sbtools') for the full example. 
\#Load non-sbtools-required but useful mapping packages

library (maps)

library (sp)

\#an item with an included OGC WFS service

layer = item_get_wfs('55e372b9e4b05561fa208212')

map ('state', regions $=$ 'iowa')

$\operatorname{plot}($ layer, add $=$ TRUE)

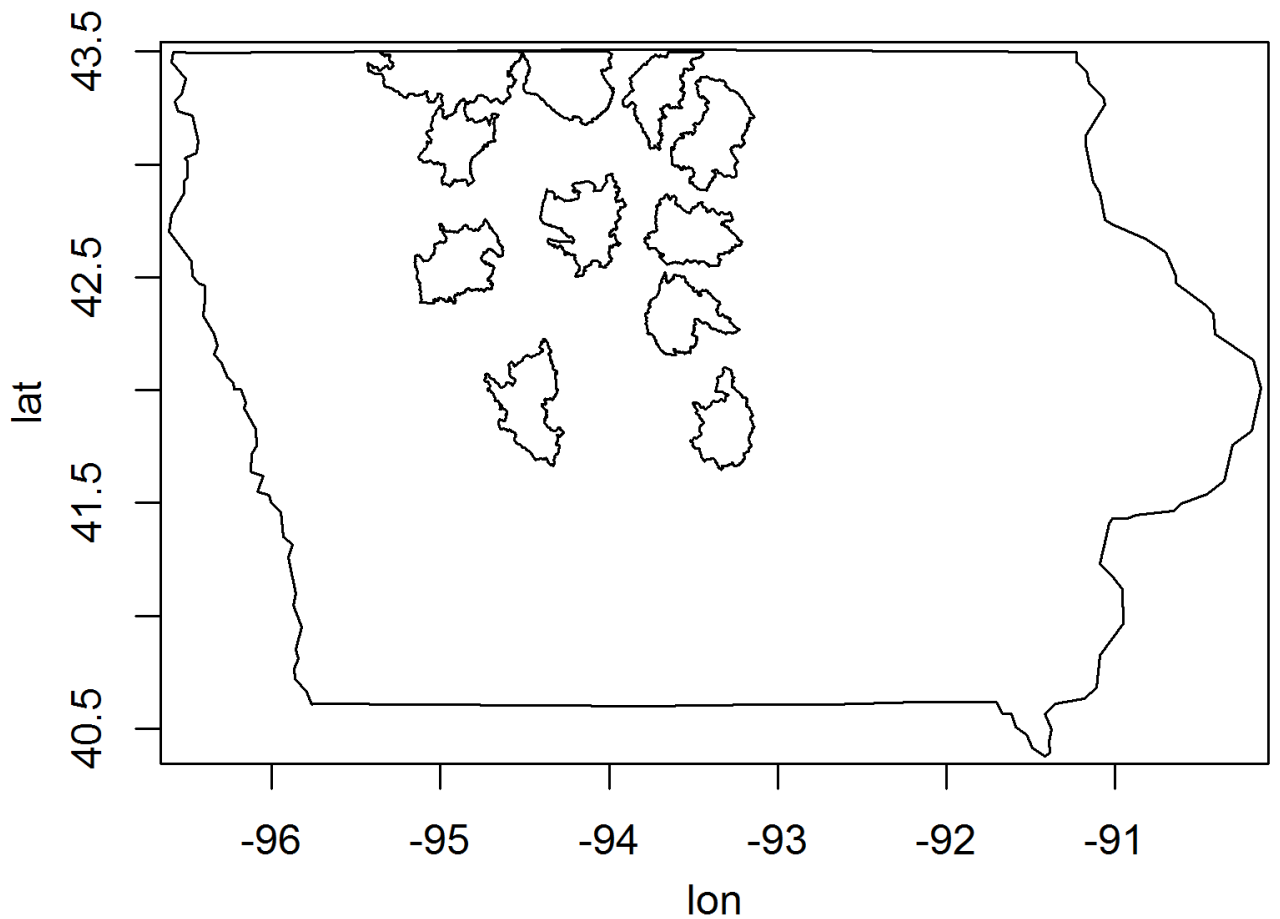

Figure 2: An example spatial dataset from ScienceBase. Shows high priority regions in Iowa containing shallow lakes and marshes to create management emphasis areas for migratory water fowl habitat.

\section{Search API}

To support advanced and powerful data discovery, all datasets in ScienceBase are indexed and made available through a flexible search interface. sbtools offers several query functions with different levels of search specificity, from cross-metadata simple text search to low-level, metadata specific search functionality.

In sbtools, query functions are included to support the most common query types. Included functions are available to search based on free-text, project folder, date-time range, geospatial bounding box, Digital Object Identifier (DOI), and data type. To save space in the examples below, we use the limit parameter to limit all queries to the first one or two results. Further details for each query type are available in the package documentation.

The ScienceBase free-text search is simple to use and generic as it searches across all of an item's text-based metadata fields. Free-text search can find specific text strings in almost all metadata fields, including but not limited to filenames, summary fields, citation, contacts, and authors. Spatial reference metadata is an example of a field not searched through the free-text search. Using sbtools, a free-text search can be run with the query_sb_text function. 


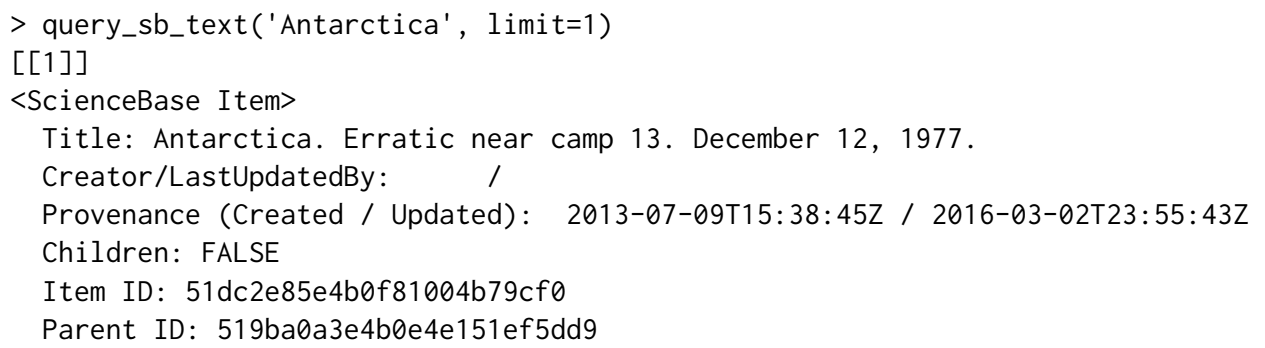

Items can also be queried according to their position in the hierarchical item tree. For example, projects funded by USGS Climate Science Centers each have sections on ScienceBase where project information is stored. Using the web interface (and some user knowledge), we first found the ScienceBase ID of the Northeast Climate Science Center community folder. Then, sbtools was used to look for specific items in that commuinty. Below is an example of looking for items containing any reference to "Lake Superior" under the Northeast Climate Science Center community.

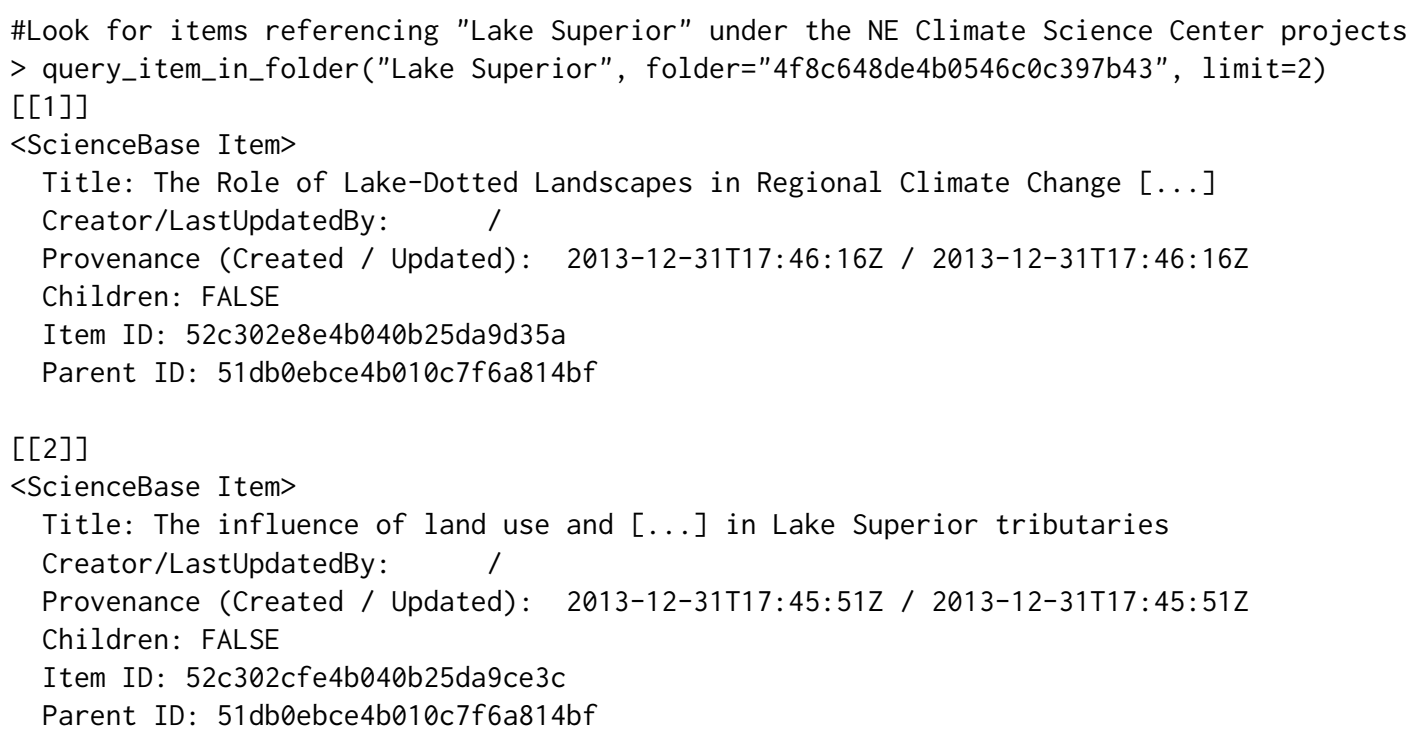

There are a several more useful query types that can be performed simply with sbtools. A few examples include date-time range, (query_sb_date ()), data type (sb_datatypes () and query_sb_datatype), and Digial Object Identifier (DOI; query_sb_doi()).

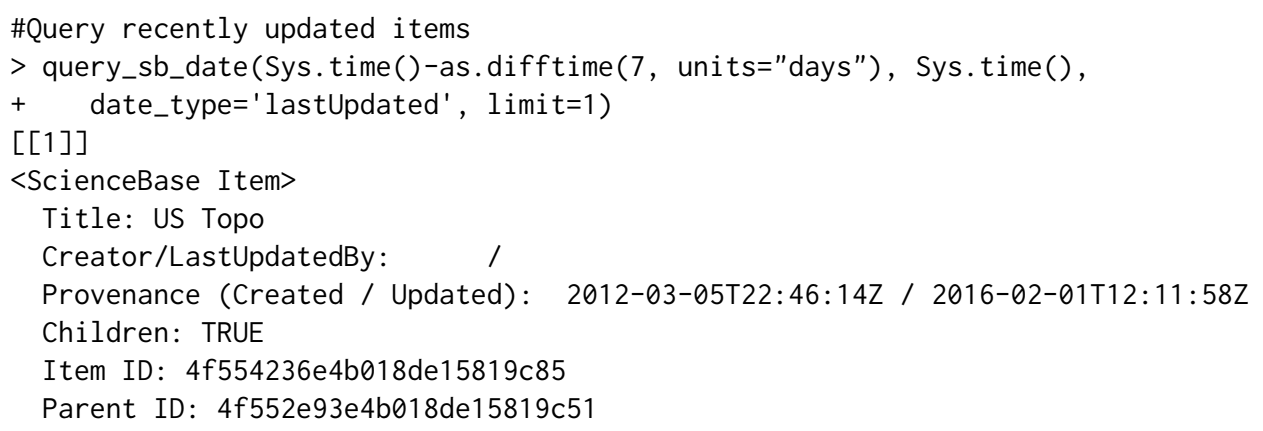


Creator/LastUpdatedBy: /

Provenance (Created / Updated): /

Children:

Item ID: 565e07b3e4b071e7ea5435d0

Parent ID: 5224e64fe4b0e4746d62af85

\#Query for DOI

$>$ query_sb_doi('10.5066/F7M043G7')

$[[1]]$

$<$ ScienceBase Item>

Title: 2013 Raw Ground Penetrating Radar Data on Alaska's Glaciers

Creator/LastUpdatedBy: /

Provenance (Created / Updated): 2015-06-15T16:55:03Z / 2015-12-15T20:39:06Z

Children: TRUE

Item ID: $557 f 0367 \mathrm{e} 4 \mathrm{~b} 023124 \mathrm{e} 8 \mathrm{ef} 621$

Parent ID: 5474ec49e4b04d7459a7eab2

Items with a geospatial component to their data or metadata can be queried using a Lat/Lon bounding box with the function query_sb_spatial(). The bounding box may be directly specified with coordinates or indirectly specified by supplying another spatial object whose bounding box should be used. Because spatial functionality requires packages beyond those imported by sbtools by default, the $\mathbf{x m 1 2}$, sp and rgdal packages must be installed to use these functions.

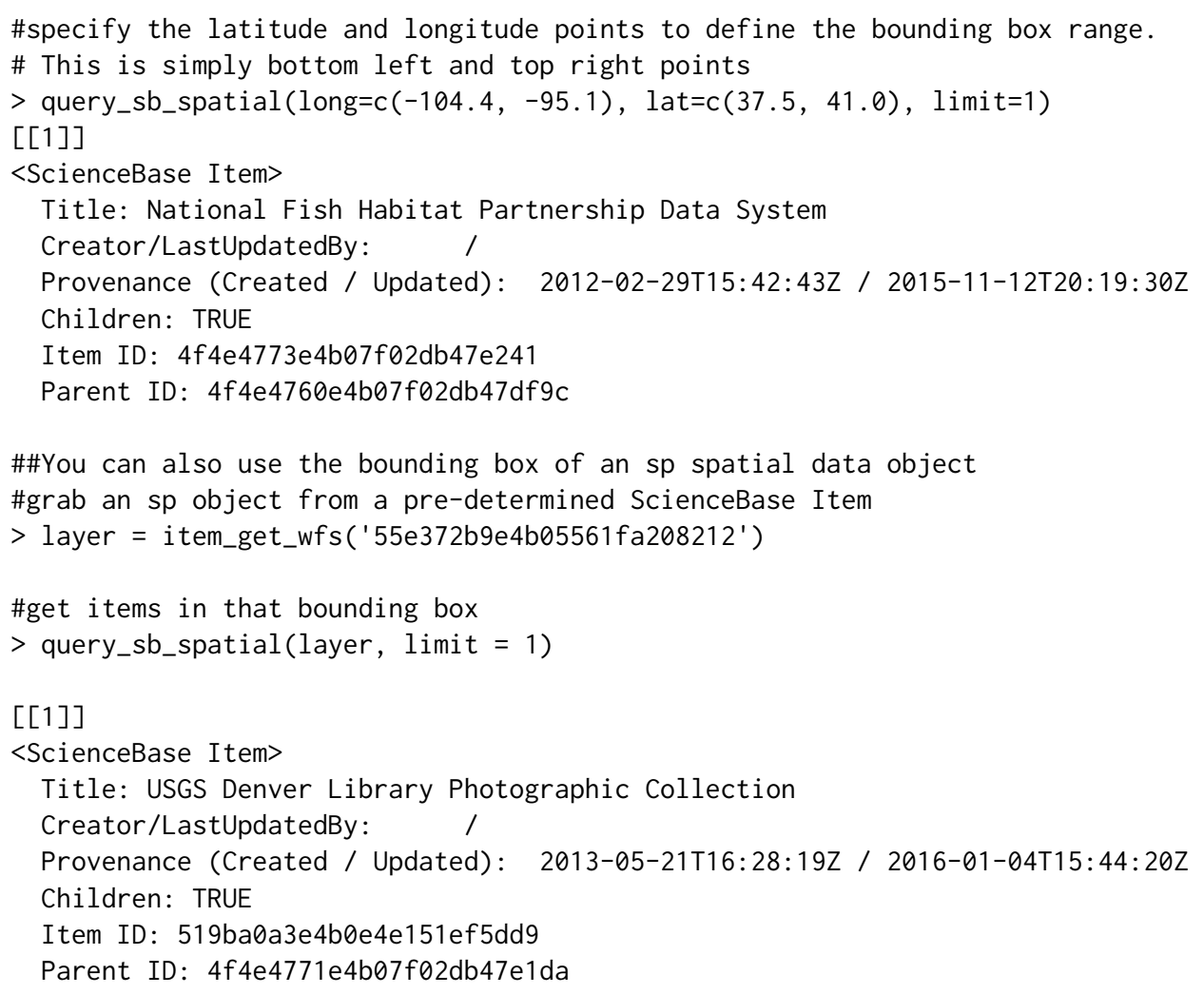

Lastly, sbtools offers advanced access to all query capabilities through the query_sb() function. query_sb() provides a convenient wrapper that allows the user to supply a list of query parameters (options described in the documentation), submits that query to ScienceBase and parses the output into a list of "sbitem" objects. When necessary, query_sb() also does proper result paging when the requested return length (limit) is over 1000 items. All advanced search options can be experimented with via the online advanced search interface.

\# query_sb can be used for combined search criteria

$>$ query_sb(list $(q=$ "water", folderId = '504216b9e4b04b508bfd337d', 
$[[1]]$

browserCategory $=$ 'Image' $)$, limit=1)

$<$ ScienceBase Item>

Title: Encyclopedia of Water Science

Creator/LastUpdatedBy: /

Provenance (Created / Updated): 2013-04-18T15:06:38Z / 2013-04-18T15:06:38Z

Children: FALSE

Item ID: 51700bfee4b05024ef3cd4ef

Parent ID: 504216b9e4b04b508bfd337d

Combining both the query functionality and direct spatial data access through WFS web services, we can quickly discover and explore available datasets on ScienceBase. For example, if we wanted to discover what faultline spatial data are available on ScienceBase, we can combine sbtools query and data access functionality. In the below example, we query for items with the word "faults" in their description which also expose data through an OGC WFS web service. We plot all datasets together to general geographic locations and data type/completeness (Figure 3). We see datasets covering much of the western United States and off the west coast. Small datasets are scattered around the gulf coast and other areas of the U.S., but none expose the raw faultline data so more discovery may be required.

Note: Some formatting details of code omitted from code below for simplicity. See demo ('figure_fault_code' , package='sbtools' ) for full example.

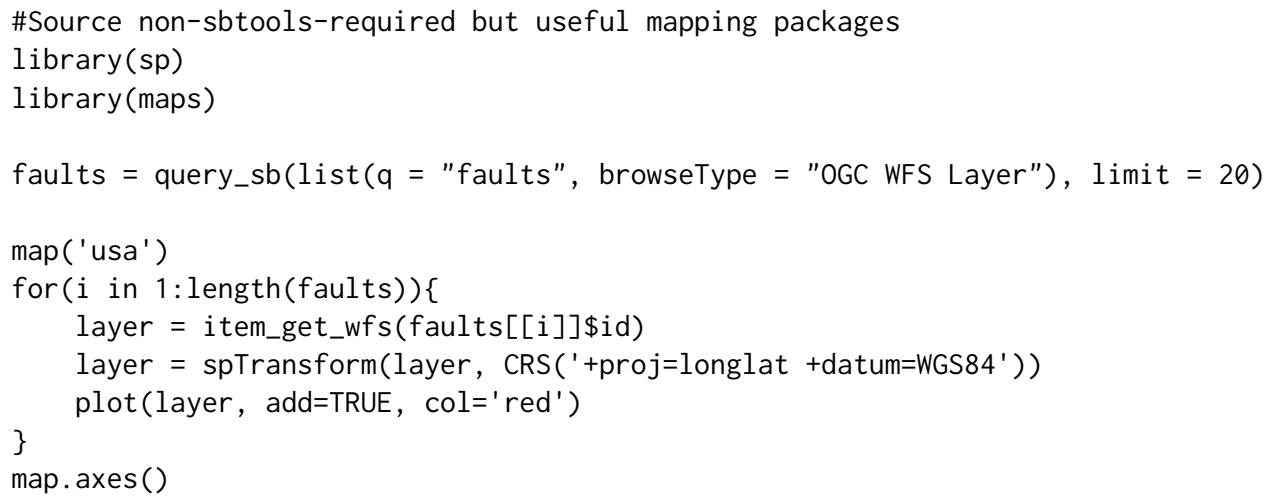

\section{ScienceBase authentication}

In addition to the large collection of open, reusable datasets and useful metadata ScienceBase has to offer, it is also a platform for sharing and collaboration on private, in-progress data available only through authenticated access. To enable private data contribution and access, sbtools has built-in support for persistent authentication of $\mathrm{R}$ sessions.

In sbtools, users can log into ScienceBase with their ScienceBase username and password using the function authenticate_sb(). To prevent plain-text passwords from being saved in the $\mathrm{R}$ command history, when using RStudio, the password can be typed into a pop-up window. The core R environment displays a terminal input interface for the password. sbtools only stores the authenticated session, thereby maintaining the confidentiality of the user's credentials. ScienceBase sessions remain active for roughly one hour and are renewed each time a request is made. sbtools makes a best effort to supply the user with meaningful error messages when a session may have expired.

\#to start an authenticated session

$>$ authenticate_sb('username@usgs.gov') \#password entered into pop-up window

$>$ is_logged_in()

[1] TRUE

$>$ session_logout()

$>$ is_logged_in()

[1] FALSE 


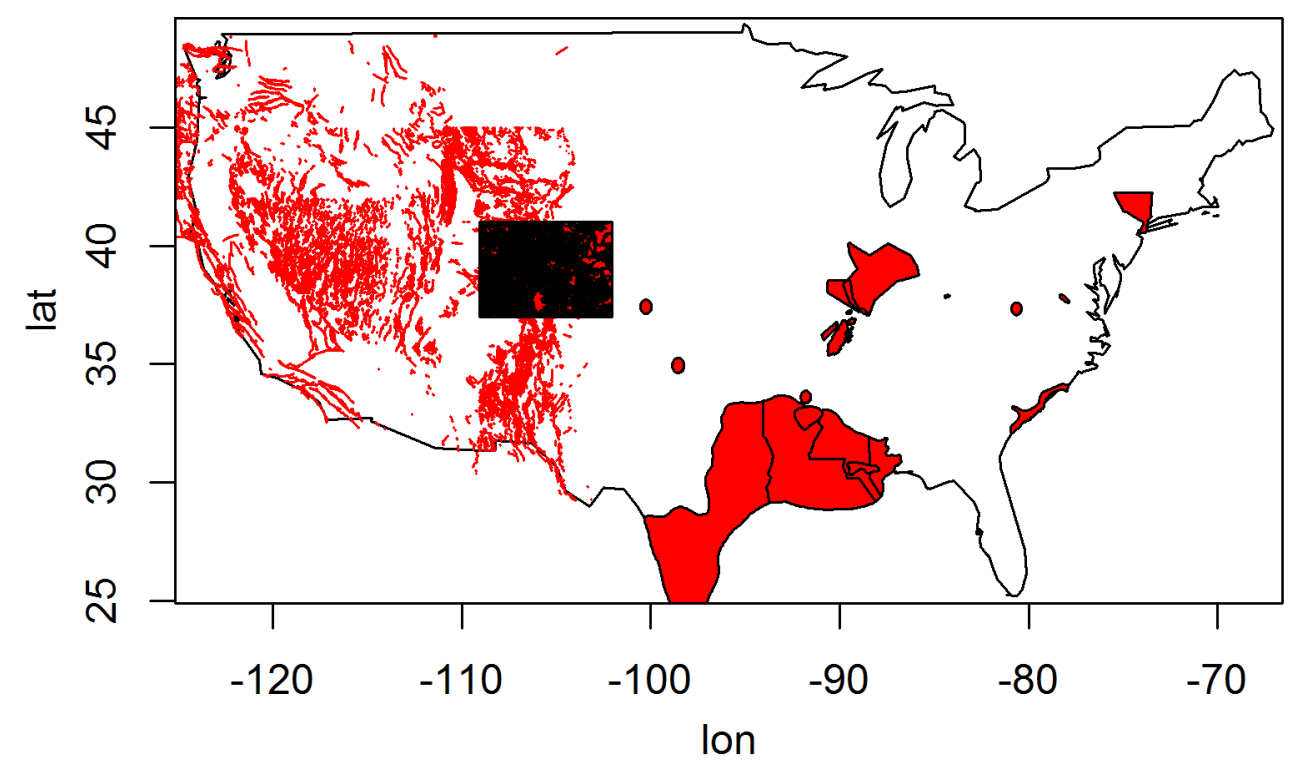

Figure 3: An example of querying and discovering multiple spatial datasets on ScienceBase. In this case, faultlines across the U.S. The variability in data formats (some lines, some polygons) is intentionally included to show both data variability, and quick discovery of different data types across various regions.

Most functions in sbtools can be used both anonymously and when authenticated. This includes all data retrieval and query functions. The behavior of these functions depends on the user's authentication status and access permissions. For example, when trying to access a private item using item_get(), you must be authenticated or you will receive an error that the item is missing. To maintain privacy, ScienceBase does not differentiate between a missing item and an item you lack permission to access. Search is also dependent on authentication status. When querying items with query_sb(), public items are always visible while private items are invisible unless authenticated.

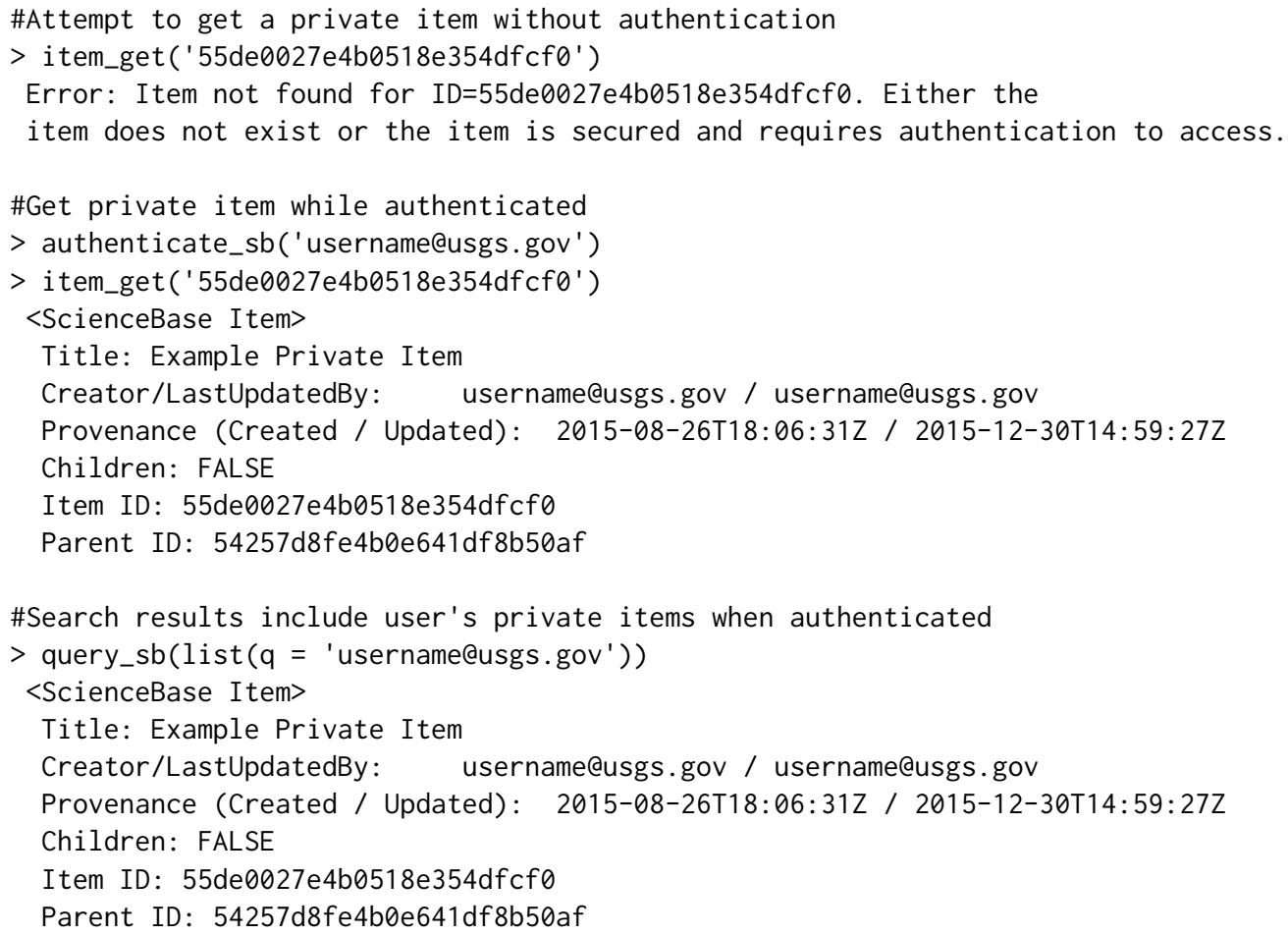

\#Search results hide private items when not authenticated $>$ session_logout() 
$>$ query_sb(list $(q=$ 'username@usgs.gov' $))$

list()

The authentication status can be quickly checked and updated with a few helper functions.

\#See if user is authenticated to SB

$>$ is_logged_in()

[1] TRUE

\#Get details of authenticated session

$>$ session_details()

\$fullDisplayName

[1] "User McUseface [username@usgs.gov]"

\$isLoggedIn

[1] TRUE

$\$$ displayName

[1] "User McUseface"

\$email

[1] "username@usgs.gov"

\$username

[1] "username@usgs.gov"

\#Renew a session to prevent expiration after 1 hour

$>$ session_renew()

\section{Data editing and upload API}

For authenticated users, sbtools can support the full data lifecycle. This includes the creation, editing and removal of items. Because item editing and creation cannot be done anonymously, this functionality only works while authenticated.

To create new items, the only required input to item_create is "title". The "Parent Item" may also be specified. New items, by default, inherit the privacy settings of their parent item. All users have a personal home folder (called "My Items" on the ScienceBase website) that serves as the default parent item for new items. The unique identifier of a user's home folder can be retrieved with the function user_id().

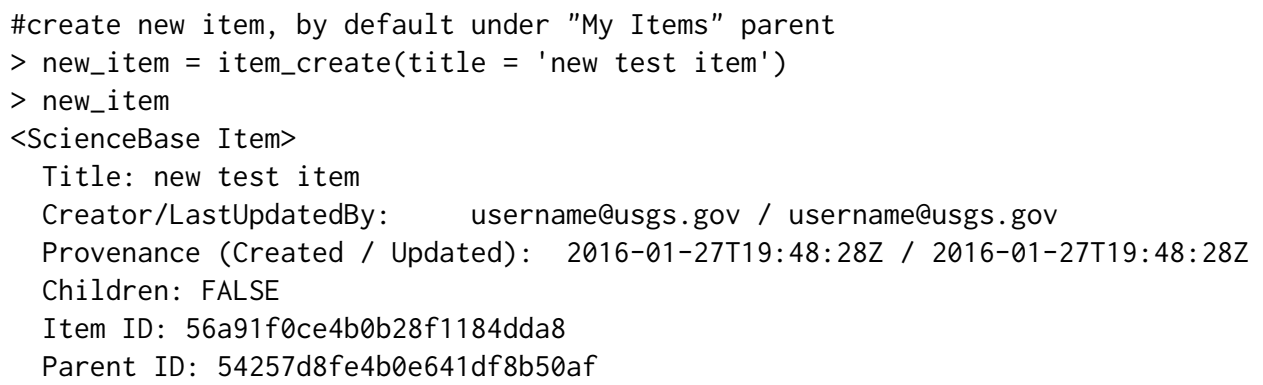

Once an item is created, an authenticated user can edit the metadata or attach data files to that item.

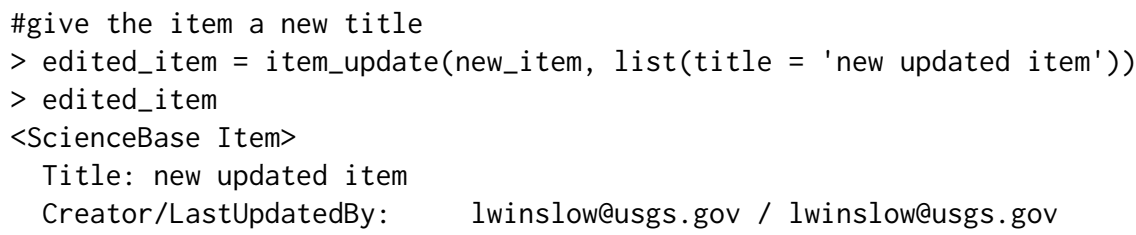




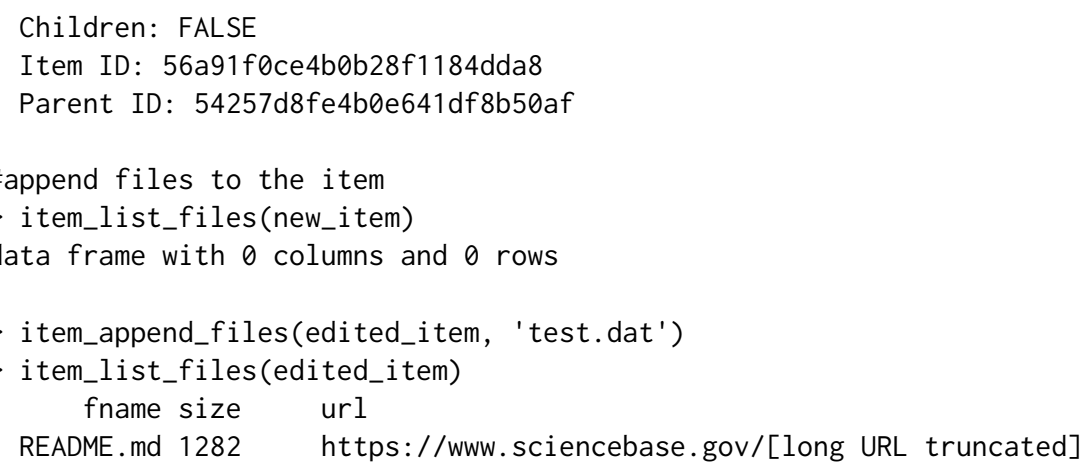

Functions are also provided to modify and delete attached files and to delete entire items.

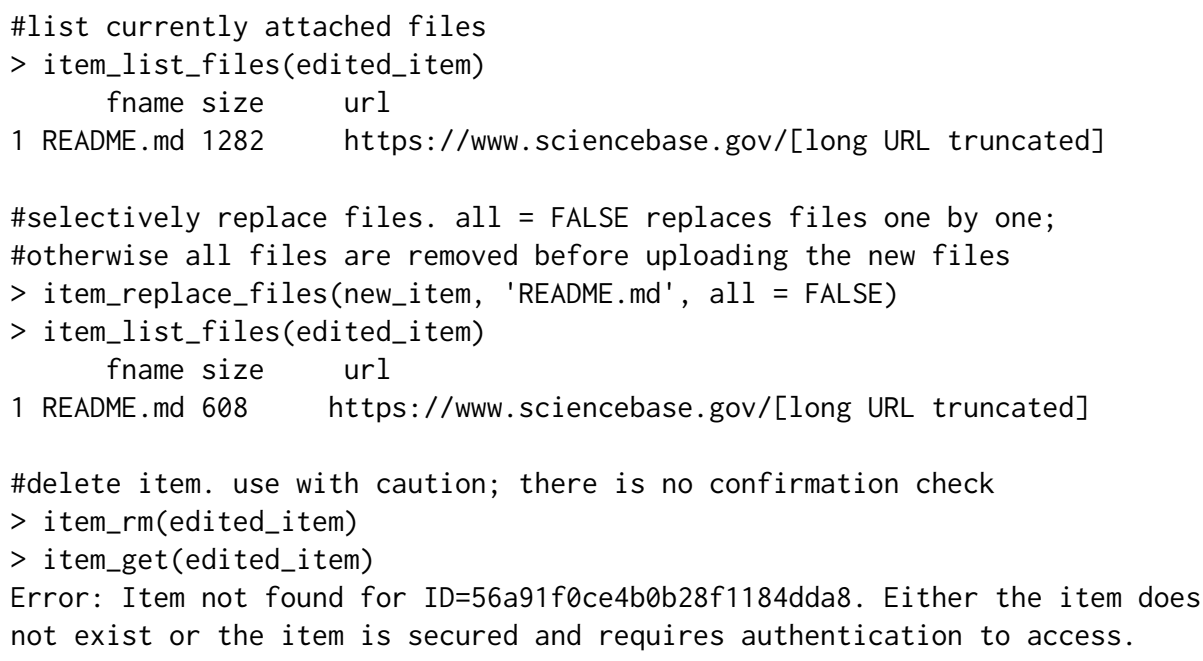

\section{SB item identifiers}

One advanced feature of ScienceBase is the ability to assign any number of custom item identifiers to items. The custom identifiers are made up of three parts: Scheme, Type and Key. Combined, these create a unique identifier. There are some standard Schemes used in ScienceBase. For example, DOIs (Digital Object Identifiers) are stored as item identifiers with the Scheme "https:/ / www.sciencebase.gov/vocab/category/item/identifier" and Type "DOI".

sbtools can edit and query custom item identifiers using item_update_identifier and query_item_identifier, respectively.

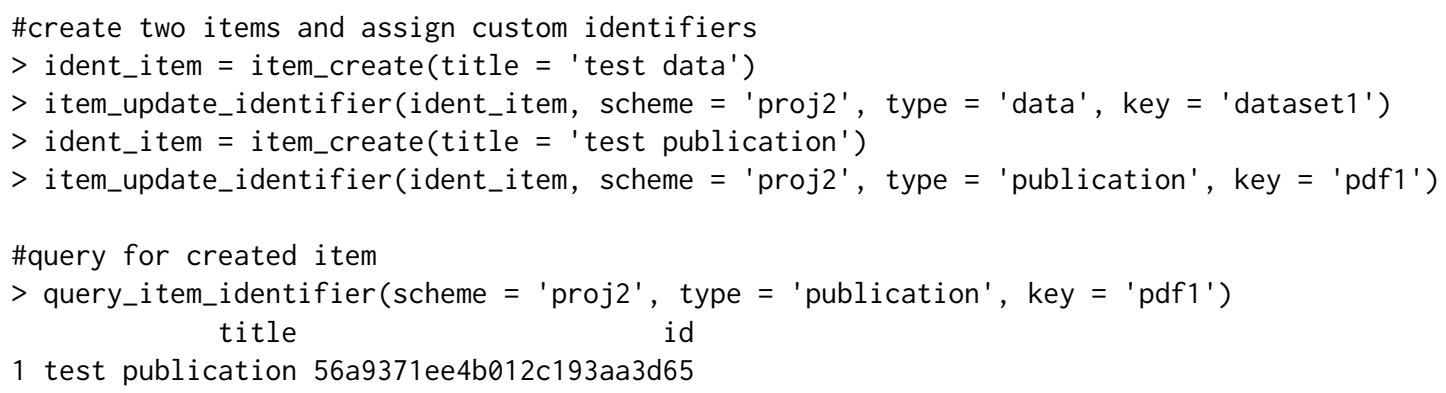

The three-part identifier can be especially useful as a way to organize and access project data. For example, all items within the same project could be created with the same scheme and differing types or keys, allowing users to query items within the project using custom tags that are meaningful to that project. 


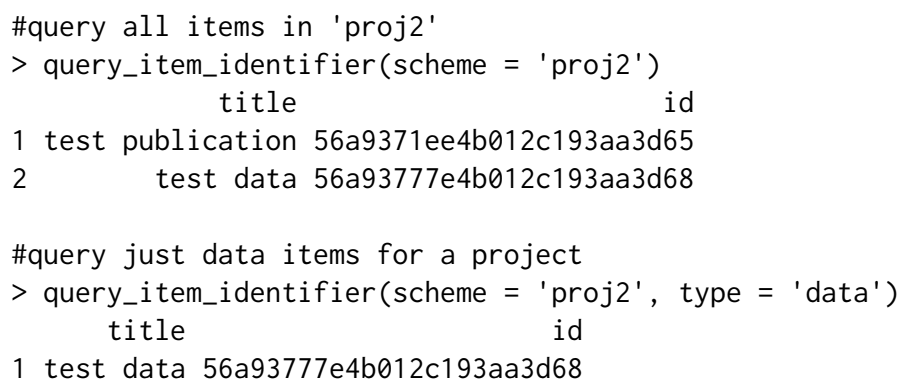

\section{Summary}

As many real-world projects have demonstrated, $\mathrm{R}$ is an excellent tool for collaborative and reproducible research projects (Gandrud, 2013a). New packages are frequently opening up access to large, open datasets (Bowman and Lees, 2015; Bowman, 2014). Flexible and powerful ways to share and collectively work with common datasets could enable new modes of collaboration and diverse data discovery. To help fill this gap, we have created sbtools. The sbtools package gives the user programmatic access to the cloud-based data and metadata storage of USGS ScienceBase and allows all researchers direct query and download capabilities to free, public data on ScienceBase. sbtools enables rapid and reproducible access to one of the single largest repositories of Earth-science data and an advanced cloud-based data collaboration platform.

\section{Acknowledgments}

This work was supported by the U.S. Geological Survey (USGS), Office of Water Information and by funding from the USGS Community for Data Integration (funding title: sbtools: An R package for ScienceBase). We would like to thank Drew Ignizio, Marian Talbert and the two journal reviewers for their careful reviews and feedback on the manuscript and package. Any use of trade, firm, or product names is for descriptive purposes only and does not imply endorsement by the U.S. Government. (

\section{Bibliography}

C. Boettiger, S. Chamberlain, K. Ram, and E. Hart. rfigshare: An R Interface to 'figshare'. URL https: //github.com/ropensci/rfigshare. R package version 0.3.7.99. [p387]

D. C. Bowman. rFDSN: Get Seismic Data from the International Federation of Digital Seismograph Networks, 2014. URL https://CRAN. R-project.org/package=rFDSN. R package version 0.0.0. [p397]

D. C. Bowman and J. M. Lees. Near real time weather and ocean model data access with rNOMADS. Computers E Geosciences, 78:88-95, 2015. doi: 10.1016/j.cageo.2015.02.013. [p397]

S. Chamberlain. ckanr: Client for the Comprehensive Knowledge Archive Network ('CKAN') 'API'. URL https://github.com/ropensci/ckanr. R package version 0.1.0. [p387]

A. Delcambre. Github - New File Size Limits, 2013. URL https://gi thub. com/blog/1533-new-filesize-limits. [p387]

C. Gandrud. Reproducible Research with R and RStudio. Chapman and Hall/CRC, Boca Raton, FL, USA, 2013a. ISBN 9781466572843. [p397]

C. Gandrud. GitHub: A tool for social data set development and verification in the cloud. SSRN Electronic Journal, 20:1-10, 2013b. ISSN 1556-5068. doi: 10.2139/ssrn.2199367. URL http://www.ssrn.com/abstract=2199367\$1delimiter"026E30F\$nhttp://papers.ssrn. com/ abstract=2199367. [p387]

M. Jones, R. Nahf, C. Jones, C. Boettiger, L. Walker, S. Chamberlain, E. Hart, J. Read, and P. Slaughter. dataone: R interface to the DataONE REST API, 2013. URL https://CRAN. R-project.org/package= dataone. R package version 1.1.0.9008. [p387] 
G. King. An Introduction to the Dataverse Network as an Infrastructure for Data Sharing. Sociological Methods E Research, 36(2):173-199, 2007. ISSN 0049-1241. doi: 10.1177/0049124107306660. [p387]

T. J. Leeper. don: Access to The Dataverse Network APIs, 2013. R package version 0.3.3. [p387]

W. K. Michener, S. Allard, A. Budden, R. B. Cook, K. Douglass, M. Frame, S. Kelling, R. Koskela, C. Tenopir, and D. A. Vieglais. Participatory design of DataONE-Enabling cyberinfrastructure for the biological and environmental sciences. Ecological Informatics, 11:5-15, 2012. ISSN 15749541. doi: 10.1016/j.ecoinf.2011.08.007. URL http://dx. doi .org/10.1016/j.ecoinf.2011.08.007. [p387]

J. Singh. FigShare. Journal of Pharmacology and Pharmacotherapeutics, 2(2):138, 2011. ISSN 0976-500X. doi: 10.4103/0976-500X.81919. URL http://www. jpharmacol.com/text. asp?2011/2/2/138/81919. [p387]

J. Winn. Open data and the academy: An evaluation of CKAN for research data management. In IASSIST 2013, Cologne, 2013. URL http://eprints. Iincoln.ac.uk/9778/. [p387]

Luke A Winslow

U.S. Geological Survey, Office of Water Information

Middleton, Wisconsin

USA

Iwinslow@usgs.gov

Scott Chamberlain

rOpenSci / University of California, Berkeley

Berkeley, California

USA

scottaropensci.org

Alison P Appling

U.S. Geological Survey, Office of Water Information

Middleton, Wisconsin

USA

aappling@usgs. gov

Jordan S Read

U.S. Geological Survey, Office of Water Information

Middleton, Wisconsin

USA

jread@usgs.gov 